\title{
Expressions diverses du potentiel électrostatique et de ses dérivées pour des systèmes à symétrie de révolution composés de cylindres de même rayon et à charge d'espace nulle
}

\author{
C. Berger \\ Laboratoire de Physique Industrielle, I.N.S.A. de Lyon \\ 69621 Villeurbanne, Cedex, France
}

M. Lambinet

I.N.S.A. de Lyon, 69621 Villeurbanne. Cedex. France

et M. Baril

Département de Physique et C.R.A.M., Université Laval, Québec, P. Qué, GlK 7P4, Canada

(Reçu le 26 avril 1979, révisé le 13 septembre 1979, accepté le 10 octobre 1979)

\begin{abstract}
Résumé. - Le but de l'étude est l'obtention pour le potentiel et ses dérivées d'expressions aussi simples que possible mettant en évidence l'influence relative des divers paramètres géométriques et électriques qui décrivent le système.

Une démarche mathématique à partir de la théorie des fonctions de Green pour le cylindre de longueur infinie conduit à des formulations comportant encore des termes où apparaissent des fonctions de Green.

L'utilisation de l'approximation mathématique de la fonction de Green par une fonction d'un cosinus hyperbolique permet d'arriver ensuite à des expressions très facilement calculables.
\end{abstract}

\begin{abstract}
The objective of the study is to obtain the simplest forms for the expression of the potential showing the influence of the various geometric and electric parameters which describe the system.

A mathematical treatment using Green function theory for infinite length cylinder result in type of formula including term showing Green function.

Mathematical approximation of Green function by hyperbolic cosine allow the transformation of the preceeding functions in easily calculable expression.
\end{abstract}

1. Introduction. - De multiples travaux se rapportent aux systèmes composés de plusieurs cylindres de même axe de révolution et de même rayon. Ces optiques ont intrinsèquement leur importance; mais par ailleurs on peut aussi ramener l'étude d'un système de révolution quelconque à celle d'un cylindre indéfini $[1,2]$.

En dehors des articles de fond $[3,4]$ auxquels nous nous référons par la suite dans le texte, ainsi que ceux de Grivet [5] et Verster [6], on rappelle ici les grandes lignes de quelques publications parmi les plus récentes en ce domaine.

Natali, Di Chiu et Kuyatt [7, 8, 9] décrivent le calcul du potentiel par la méthode des réseaux et celui des trajectoires, puis ils déterminent les propriétés optiques et les coefficients d'aberration d'une lentille bicylindrique.
A la même période, Read, Adams et Soto-Montiel $[10,11,12,13]$ publient de multiples données sur les lentilles composées de deux et trois cylindres, le champ est calculé par sommation numérique des contributions de petits cylindres.

Plus récemment Gallaï et Szilágyi [14] s’intéressent à la distribution de potentiel dans un système présentant des propriétés de périodicité. La mesure du potentiel en un nombre $K$ de points entre les cylindres permet d'approcher le potentiel aux limites dans l'intervalle interélectrode par une fonction d'ordre $2 K-1$. Le potentiel est alors obtenu en un point quelconque par une sommation faisant intervenir les fonctions de Bessel.

Enfin tout dernièrement Bonjour $[15,16]$ donne les caractéristiques optiques et les coefficients d'aberration pour des lentilles à deux ou trois cylindres en 
déterminant le champ à partir d'une méthode décrite par Bertram [17] utilisant l'expression de l'énergie potentielle avec une approximation du troisième ordre pour le potentiel aux limites.

En dehors de la méthode des réseaux dont on ne peut attendre qu'une précision limitée, toutes les méthodes proposées font appel à des sommations plus ou moins complexes. Ces modes de calcul nécessitent donc tous des temps de calcul assez longs. De plus il est impossible de déduire de ces formules la contribution individuelle exacte de chaque paramètre géométrique ou électrique. Toute entreprise d'amélioration de la qualité optique de ces systèmes s'avère donc une tâche difficilement réalisable en utilisant de tels processus.

Le but que nous nous sommes fixé ici est double. Il est de trouver des expressions analytiques plus simples pour le potentiel et de mettre en évidence l'influence de chacun des paramètres géométriques et électriques.

1. Analyse du problème. - 1.1 DESCRIPTION DES SYSTÈMES ÉTUDIÉS. - Les systèmes étudiés ici doivent avoir les deux caractéristiques suivantes :

- ne pas présenter de charges d'espace,

- être équivalents à un cylindre de révolution de longueur infinie avec répartition de révolution du potentiel connue en tout point.

Deux catégories de systèmes répondent à cette description.

Le premier type de systèmes est constitué d'un ensemble de cylindres de même axe et de même rayon, ceux des extrémités ayant des longueurs suffisamment grandes pour être supposés infinis. Un exemple en est donné à la figure 1 .

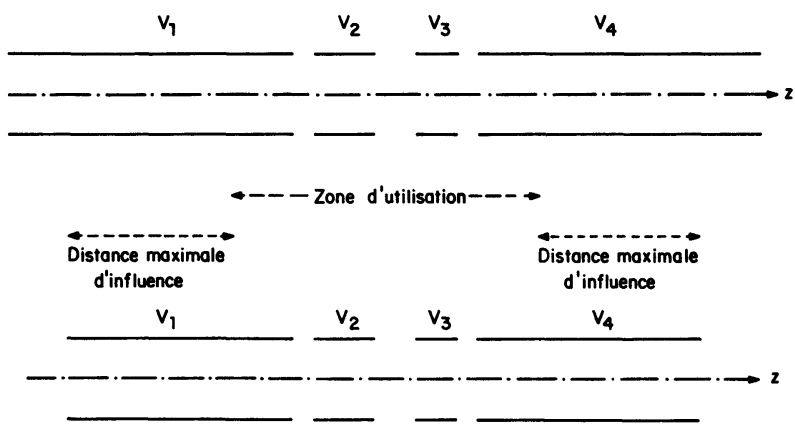

Fig. 1. - Transformation d'un système de longueur infinie en un système de longueur finie.

[Transformation of an infinite length system into a finite length system.]

Dans les calculs, si cela est nécessaire, ces systèmes peuvent être considérés comme ayant des longueurs plus réduites en supprimant les portions situées audelà de la distance maximale d'influence, évaluée à cinq fois le rayon du cylindre.
Le deuxième type de systèmes répondant à cette étude est représenté par la figure $2 a$. Il s'agit d'un ensemble de cylindres fermés par deux diaphragmes dont les plans sont supposés équipotentiels dans leur totalité.

Un décalage $-V_{c}$ du potentiel des électrodes crée en tout point le même décalage des potentiels mais les valeurs des dérivées sont inchangées. On peut donc étudier le système représenté sur la figure $2 b$.

Il peut être remplacé par le système équivalent de la figure $2 c$, compte tenu de l'antisymétrie par rapport au plan $\left(\mathbf{P}_{1}\right)$.
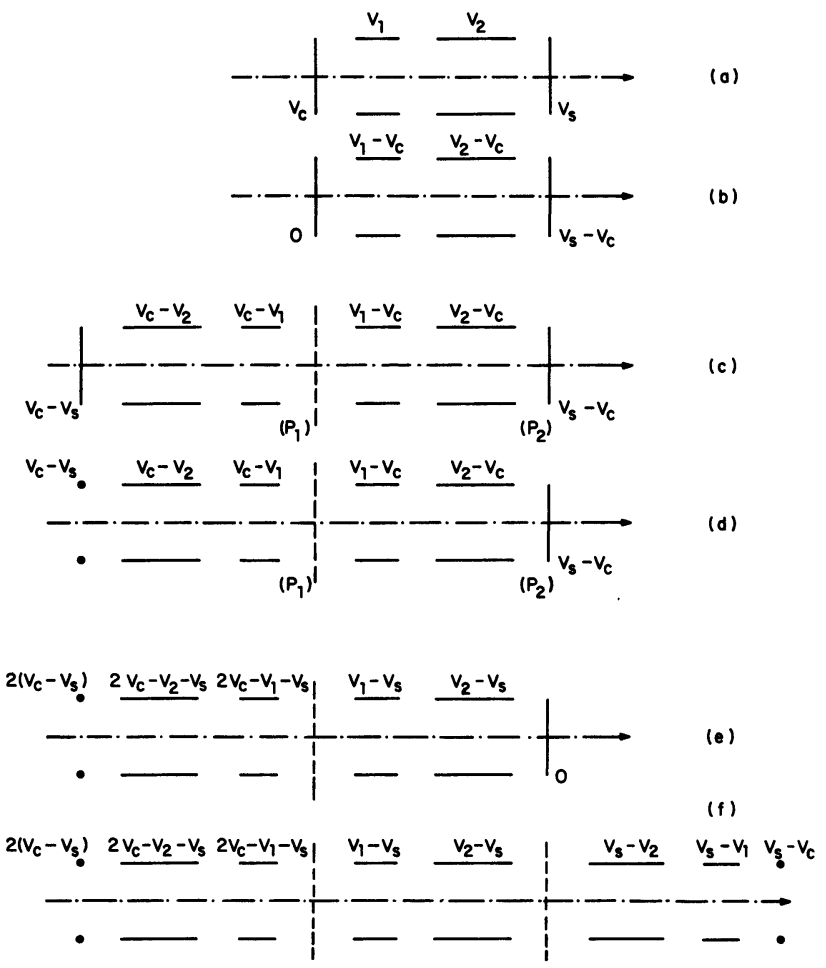

Fig. 2. - Transformations successives du système représenté en (a) en vue d'une simplification de son étude.

[Successive transformation of the system shown in (a) to simplify its study.]

En supposant la distance $\left(P_{1}\right)-\left(P_{2}\right)$ supérieure à la distance maximale d'influence, il est possible de remplacer le système par celui de la figure $2 d$.

Un nouveau décalage des potentiels conduit au système de la figure $2 e$, équivalant pour la portion comprise entre $\left(\mathrm{P}_{1}\right)$ et $\left(\mathrm{P}_{2}\right)$ au système de la figure $2 f$.

Quelle que soit la distance $\left(P_{1}\right)-\left(P_{2}\right)$ moyennant un certain nombre de décalages de potentiel et de transformations dues à l'antisymétrie, un système de ce deuxième type peut toujours être ramené à un cylindre de longueur infinie avec une distribution de révolution des potentiels.

1.2 DÉCOMPOSITION EN SYSTÈMES ÉLÉMENTAIRES. L'étude à réaliser est donc celle d'un cylindre de révolution de longueur infinie dont on connaît la réparti- 


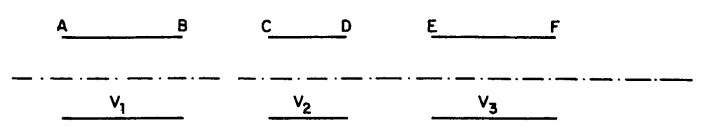

Fig. 3. - Exemple de répartition de potentiel le long d'un cylindre de révolution de longueur infinie.

[Example of potential distribution along a circular cylinder of infinite length.]

tion du potentiel. La figure 3 en présente un exemple particulier.

Du point de vue électrique ce système est équivalent à la superposition d'un certain nombre de cylindres de longueur infinie, de rayon identique à celui du système, chaque cylindre représentant la contribution d'une électrode ou d'un espacement.

Par exemple, le potentiel du cylindre associé à l'électrode $\mathrm{AB}$ est nul partout sauf sur l'emplacement $\mathrm{AB}$ où sa valeur est $V_{1}$ potentiel de l'électrode. Le potentiel du cylindre associé à l'espacement $\mathrm{BC}$ est nul partout sauf sur l'emplacement $\mathrm{BC}$ où il varie linéairement de $V_{1}$ à $V_{2}$.

Pour l'évaluation de sa contribution, ce dernier type de cylindre est considéré comme la superposition de deux cylindres de longueur infinie où le potentiel est nul partout sauf entre B et C. Pour l'un, le potentiel entre $\mathrm{B}$ et $\mathrm{C}$ est constant et vaut $\frac{V_{1}+V_{2}}{2}$, pour lautre il varie linéairement de $-\frac{V_{2}-V_{1}}{2}$ à $+\frac{V_{2}-V_{1}}{2}$.

En conclusion l'étude des systèmes peut, compte tenu de l'emploi du théorème de superposition, être ramenée à celle des deux systèmes élémentaires décrits avec précision dans le paragraphe 1.3.2.

1.3 Principe de CAlCUl DU Potentiel et DE SES DÉRIVÉES. - 1.3.1 Fonction de Green pour un cylindre indéfini. - Dans le cas d'un cylindre indéfini (Fig. 4) le potentiel en un point $M(z)$ de l'axe, s'exprime en fonction du potentiel des différents points $P(\zeta)$ de la surface du cylindre :

$$
V(z)=\int_{-\infty}^{+\infty} V(\zeta) G(\zeta, z) \mathrm{d} \zeta
$$

$G(\zeta, z)$ étant la fonction de Green du cylindre indéfini pour le couple de points $(\mathrm{P}, \mathrm{M})$, soit :

$$
G(\zeta, z)=\frac{1}{a} \sum_{i=1}^{\infty}\left\{\frac{1}{J_{1}\left(m_{i} a\right)} \exp \left[-m_{i}|\zeta-z|\right]\right\}
$$

où :

$a$ est le rayon du cylindre,

$m_{i}$ est racine de l'équation $J_{0}\left(m_{i} a\right)=0, J_{0}$ étant

la fonction de Bessel de première espèce d'ordre 0 ,

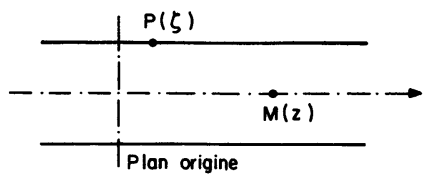

Fig. 4. - Système formé d'un cylindre indéfini.

[System made of a cylinder of infinite length.]

$J_{1}$ est la fonction de Bessel de première espèce d'ordre 1.

Lorsque $|\zeta-z|$ est petit, la convergence est très lente; il est alors préférable d'utiliser l'expression suivante :

$$
G(\zeta, z)=\frac{1}{\pi} \int_{0}^{\infty} \frac{1}{I_{0}(k a)} \cos [k(\zeta-z)] \mathrm{d} k
$$

où : $I_{0}$ est la fonction de Bessel modifiée de première espèce d'ordre 0.

1.3.2 Expressions du potentiel sur l'axe des deux systèmes élémentaires. - D’après le paragraphe 1.2, le problème revient à étudier les deux types suivants de répartition du potentiel sur un cylindre de longueur infinie.

Pour le premier système nommé ÉLECTRODE les conditions sur le cylindre sont :

$$
\begin{aligned}
& V(\zeta)=1 \text { pour } \zeta \in]-\frac{L}{2}, \frac{L}{2}[ \\
& V(\zeta)=0 \text { pour } \zeta \notin\left[-\frac{L}{2}, \frac{L}{2}\right] .
\end{aligned}
$$

Le potentiel sur l'axe $a$ a alors pour expression

$$
V_{\mathrm{e}}(z, L)=\int_{-L / 2}^{+L / 2} G(z-\zeta) \mathrm{d} \zeta .
$$

Pour le deuxième système nommé INTERVALLE les conditions sur le cylindre sont :

$$
\begin{aligned}
& \left.V(\zeta)=\frac{\zeta}{L} \text { pour } \zeta \in\right]-\frac{L}{2}, \frac{L}{2}[ \\
& V(\zeta)=0 \quad \text { pour } \zeta \notin\left[-\frac{L}{2}, \frac{L}{2}\right] .
\end{aligned}
$$

Le potentiel sur laxe $a$ a alors pour expression

$$
V_{i}(z, L)=\frac{1}{L} \int_{-L / 2}^{L / 2} \zeta G(z-\zeta) \mathrm{d} \zeta .
$$

Le rayon du cylindre est choisi comme unité de longueur afin de simplifier les calculs.

2. Formulations proposées pour les deux systèmes élémentaires. - On désignera par $G_{n}$ la dérivée $n$-ième de $G$ (fonction d'une variable); $G_{0}$ désignant $G$ 
elle-même, la notation sera valable quel que soit $n$ entier naturel.

\subsection{CalCul EXACT DES DÉRIVÉES PAR RAPPORT A $z$.}

$-1 \mathrm{er}^{\mathrm{e}}$ cas : ÉLECTRODE.

$$
V_{\mathrm{e}}(z, L)=\int_{-L / 2}^{+L / 2} G(\zeta-z) \mathrm{d} \zeta
$$

Le théorème de dérivation sous le signe somme donne

$$
\frac{\partial V_{\mathrm{e}}}{\partial z}=\int_{-L / 2}^{+L / 2} \frac{\partial G(\zeta-z)}{\partial z} \mathrm{~d} \zeta
$$

d'où

$$
\frac{\partial V_{\mathrm{e}}}{\partial z}=G\left(z+\frac{L}{2}\right)-G\left(z-\frac{L}{2}\right) .
$$

En dérivant encore $(n-1)$ fois par rapport à $z$

$$
\frac{\partial^{n} V_{\mathrm{e}}(z, L)}{\partial z_{n}}=G_{n-1}\left(z+\frac{L}{2}\right)-G_{n-1}\left(z-\frac{L}{2}\right)
$$

2e cas : INTERVALLE.

$$
V_{i}(z, L)=\int_{-L / 2}^{+L / 2} \zeta G(\zeta-z) \mathrm{d} \zeta .
$$

La dérivation par rapport à $z$ sous le signe somme et une intégration par partie conduisent à :

$$
\begin{aligned}
\frac{\partial V_{i}}{\partial z}=-\frac{1}{L}[\zeta G(\zeta-z)]_{\zeta=-L / 2}^{L / 2} & \\
& +\frac{1}{L} \int_{-L / 2}^{+L / 2} G(\zeta-z) \mathrm{d} \zeta
\end{aligned}
$$

La dernière intégrale n'est autre que $V_{\mathrm{e}}$; d'où compte tenu de la parité de $G$

$$
\begin{aligned}
& \frac{\partial V_{i}(z, L)}{\partial z}= \\
& \quad=-\frac{1}{2}\left[G\left(z+\frac{L}{2}\right)+G\left(z-\frac{L}{2}\right)\right]+\frac{V_{\mathrm{e}}(z, L)}{L} .
\end{aligned}
$$

En dérivant $(n-1)$ fois par rapport à $z$

$$
\begin{aligned}
\frac{\partial V_{i}^{n}(z, L)}{\partial z^{n}}= & -\frac{1}{2}\left[G_{n-1}\left(z+\frac{L}{2}\right)+G_{n-1}\left(z-\frac{L}{2}\right)\right] \\
& +\frac{1}{L}\left[G_{n-2}\left(z+\frac{L}{2}\right)-G_{n-2}\left(z-\frac{L}{2}\right)\right]
\end{aligned}
$$

2.2 EXPRESSIONS NE FAISANT PLUS INTERVENIR LES FONCTIONS DE GREEN. - 2.2.1 Approche analytique de la fonction $G(z)$. - Dans le cas d'un système formé de deux cylindres de très grande longueur, juxtaposés avec un espacement négligeable, comme le montre la figure 5 , le potentiel $V$ sur l'axe est bien décrit par

$$
V \simeq \frac{V_{1}+V_{2}}{2}+\frac{V_{2}-V_{1}}{2} \text { th } \omega z_{0}
$$

$V_{1}$ est le potentiel du $1^{\mathrm{er}}$ cylindre (à l'intérieur duquel se meuvent les particules),

$V_{2}$ le potentiel du deuxième cylindre;

$z_{0}$ l'abscisse du point de potentiel $V$, repérée par rapport à la séparation des deux cylindres :

$\omega$ une constante.

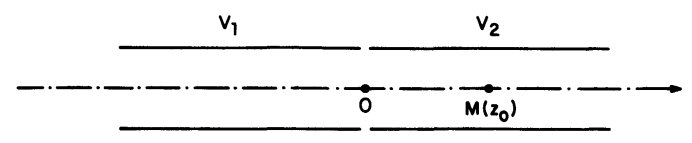

Fig. 5. - Cylindres juxtaposés avec espacement négligeable.

[Two neighbouring cylinders with negligible spacing in between.]

La dérivée première du potentiel a pour expression :

$$
\frac{\partial V}{\partial z}=\frac{V_{2}-V_{1}}{2} \cdot \frac{\omega}{\operatorname{ch}^{2} \omega z_{0}} .
$$

L'utilisation des formules exactes du 2.2 pour le calcul de la dérivée première conduit à

$$
\frac{\partial V}{\partial z}=\left(V_{2}-V_{1}\right) G\left(z_{0}\right)
$$

La comparaison des deux expressions de $\partial V / \partial z$ donne

$$
G(z) \simeq \frac{1}{2} \frac{\omega}{\operatorname{ch}^{2} \omega z}
$$

De cette dernière formule, on en déduit

$$
\omega=2 G(0)=1,32619
$$

valeur en accord avec celle donnée par Verster [6] soit $\omega=1,32623$, légèrement différente de la valeur 1,318 habituellement utilisée.

2.2.2 Utilisation de l'expression de G. - Dans les développements proposés au $2.1, G(z)$ et ses dérivées peuvent être remplacées par des fonctions plus simples à calculer.

Mais cette expression de $G$ va être particulièrement intéressante dans les formules exactes du 2.1.

1 er cas : ÉLECTRODE.

La formule

$$
\frac{\partial V_{\mathrm{e}}(z, L)}{\partial z}=G\left(z+\frac{L}{2}\right)-G\left(z-\frac{L}{2}\right)^{\prime}
$$


devient

$\frac{\partial V_{\mathrm{e}}(z, L)}{\partial z}=\frac{1}{2}\left[\frac{\omega}{\operatorname{ch}^{2} \omega\left(z+\frac{L}{2}\right)}-\frac{\omega}{\operatorname{ch}^{2} \omega\left(z-\frac{L}{2}\right)}\right]$

Par intégration, on obtient le potentiel

$$
\begin{aligned}
V_{\mathrm{e}}(z, L) & \simeq \\
\simeq & \frac{1}{2}\left[\operatorname{th} \omega\left(z+\frac{L}{2}\right)-\operatorname{th} \omega\left(z-\frac{L}{2}\right)\right]+\text { Cte } .
\end{aligned}
$$

Le potentiel étant nul à l'infini, la constante est nulle.

$$
V_{\mathrm{e}}(z, L) \simeq \frac{1}{2}\left[\operatorname{th} \omega\left(z+\frac{L}{2}\right)-\operatorname{th} \omega\left(z-\frac{L}{2}\right)\right]
$$

$2^{\mathrm{e}}$ cas : INTERVALLE.

On part de :

$$
\begin{aligned}
& \frac{\partial V_{i}(z, L)}{\partial z}= \\
& \quad=-\frac{1}{2}\left[G\left(z+\frac{L}{2}\right)+G\left(z-\frac{L}{2}\right)\right]+\frac{V_{\mathrm{e}}(z, L)}{L}
\end{aligned}
$$

et l'on remplace les fonctions par leurs expressions en fonction des lignes hyperboliques

$$
\begin{aligned}
\frac{\partial V_{i}(z, L)}{\partial z} \simeq-\frac{1}{4}\left[\frac{\omega}{\operatorname{ch}^{2} \omega\left(z+\frac{L}{2}\right)}+\frac{\omega}{\operatorname{ch}^{2} \omega\left(z-\frac{L}{2}\right)}\right]+ \\
+\frac{1}{2 L}\left[\operatorname{th~} \omega\left(z+\frac{L}{2}\right)-\operatorname{th} \omega\left(z-\frac{L}{2}\right)\right]
\end{aligned}
$$

en intégrant par rapport à $z$, on a

$$
\begin{array}{r}
V_{i}(z, L) \simeq-\frac{1}{4}\left[\operatorname{th} \omega\left(z+\frac{L}{2}\right)+\operatorname{th} \omega\left(z-\frac{L}{2}\right)\right]+ \\
+\frac{1}{2 L \omega} \ln \frac{\operatorname{ch} \omega\left(z+\frac{L}{2}\right)}{\operatorname{ch} \omega\left(z-\frac{L}{2}\right)}+\text { Cte } .
\end{array}
$$

\begin{tabular}{|c|c|c|c|c|c|c|c|}
\hline & $z$ & $\underline{G_{0}}$ & $G_{1}$ & $G_{2}$ & $G_{3}$ & $G_{4}$ & $\underline{G_{5}}$ \\
\hline F. normale & & 0,663095 & 0,0 & $-2,49477$ & 0,0 & 40,3977 & 0,0 \\
\hline \multirow{2}{*}{ F. approchée $(*)$} & $\int 0,00$ & 0,663096 & 0,0 & $-2,33249$ & 0,0 & 32,8187 & 0,0 \\
\hline & & 0,659175 & 0,0 & $-2,29135$ & 0,0 & 31,8598 & 0,0 \\
\hline F.N. & 0,25 & 0,591242 & $-0,529320$ & $-1,44162$ & 6,95554 & 7,25272 & $-183,700$ \\
\hline & & 0,595233 & $-0,505071$ & - 1,45094 & 6,01554 & 10,20606 & $-141,620$ \\
\hline $\begin{array}{l}\text { F.N. } \\
\text { F.A. }\end{array}$ & 0,50 & $\begin{array}{l}0,431853 \\
0,439707\end{array}$ & $\begin{array}{l}-0,678969 \\
-0,676928\end{array}$ & $\begin{array}{l}0,138389 \\
0,016492\end{array}$ & $\begin{array}{l}4,60626 \\
4,71151\end{array}$ & $\begin{array}{l}-18,4080 \\
-14,6996\end{array}$ & $\begin{array}{l}-12,1761 \\
-\quad 31,7260\end{array}$ \\
\hline F. normale & & 0,275490 & $-0,546577$ & 0,754188 & 0,666116 & $-10,7927$ & 45,9987 \\
\hline F. approchée $(*)$ & $\{0,75$ & $\begin{array}{l}0,280761 \\
0,281601\end{array}$ & $\begin{array}{l}-0,565467 \\
-0,561947\end{array}$ & $\begin{array}{l}0,720720 \\
0,703215\end{array}$ & $\begin{array}{l}1,07501 \\
1,10017\end{array}$ & $\begin{array}{l}-11,5474 \\
-11,3683\end{array}$ & $\begin{array}{l}36,8709 \\
35,3561\end{array}$ \\
\hline F.N. & & 0,162742 & $-0,358402$ & 0,690923 & $-0,806500$ & $-2,06715$ & 21,5304 \\
\hline F.A. & 1,00 & 163140 & $-0,375729$ & 0,724160 & $-0,69$ & 3,15898 & 24,7048 \\
\hline F.N. & 1,50 & 0,051516 & $-0,121618$ & 0,280107 & $-0,606743$ & 1,10175 & 0,776305 \\
\hline F.N & & 0,015653 & $-0,037497$ & 0.089369 & $-0,210493$ & 0,481939 & $\begin{array}{l}0,212647 \\
1.02647\end{array}$ \\
\hline F.A. & 2,00 & 0,013047 & $-0,034263$ & 0,089076 & $-0,226815$ & 0,552311 & 1,21007 \\
\hline F.N. & 2,50 & 0,004714 & $-0,011328$ & 0,027191 & $-0,065107$ & 0,155013 & 0,364198 \\
\hline F.A. & 2,00 & 0,003489 & $-0,009230$ & 0,024352 & & 0,165904 & 0,421044 \\
\hline F.N. & 3,00 & 0,001417 & $-0,003407$ & 0,008192 & $-0,019668$ & 0,047231 & 0,113037 \\
\hline & 3,00 & 0,000928 & $-0,002460$ & 0,006515 & $-0,017233$ & 0,045452 & 0,119198 \\
\hline
\end{tabular}

En écrivant que $V=0$ pour $z=0$, on en déduit

$$
\begin{array}{r}
V_{i}(z, L) \simeq-\frac{1}{4}\left[\operatorname{th} \omega\left(z+\frac{L}{2}\right)+\operatorname{th} \omega\left(z-\frac{L}{2}\right)\right]+ \\
+\frac{1}{2 L \omega} \ln \frac{\operatorname{ch} \omega\left(z+\frac{L}{2}\right)}{\operatorname{ch} \omega\left(z-\frac{L}{2}\right)}
\end{array}
$$

Tableau I. - Comparaison des valeurs de la fonction de Green et de ses dérivées obtenues par la formule normale et par la formule approchée.

[Comparison of the values of Green function and their derivatives obtained by normal and approximative formulas.]

$\left({ }^{*}\right)$ Sur les lignes marquées d'un astérisque, la valeur prise pour $\omega$ est 1,31835 ; partout ailleurs les calculs ont été faits avec $\omega=1,32619$. 
2.2.3 Limite de validité de ces expressions. A. El-Kareh et J. El-Kareh présentent une étude chiffrée des erreurs introduites par le remplacement de

$$
\int_{0}^{x} \sin k z \cdot \frac{1}{J_{0}(i k)} \mathrm{d} k \text { par } \frac{\pi}{2} \text { th } \omega z .
$$

L'erreur relative est en général bien inférieure à $1 \%$, sa valeur maximale est évaluée à $0,85 \%$ pour $z=1$. On peut donc attendre une précision très acceptable des valeurs du potentiel calculées par les formules approchées proposées.

Par ailleurs, le tableau I permet la comparaison des valeurs de la fonction de Green et de ses dérivées calculées par l'expression normale à celles obtenues par l'expression approchée. De plus pour $z=0$ et $z=0,75$ les valeurs approchées ont également été évaluées en prenant $\omega=1,31835$, pour montrer que l'approximation avec $\omega=1,32619$ est en général meilleure.

Pour $G$ et ses trois premières dérivées $G_{1}, G_{2}, G_{3}$ même si l'erreur relative devient parfois importante, l'erreur vraie reste toujours faible car cela se produit pour des valeurs quasi négligeables des fonctions. Ces conclusions coïncident avec celles des El-Kareh, déduites de la comparaison des deux fonctions citées plus haut.

Au fur et à mesure de la dérivation des fonctions $G$, l'approximation devient de moins en moins valable, si bien que pour $G_{4}$ et $G_{5}$ les formules proposées ne fournissent qu'un ordre de grandeur.

La connaissance des dérivées du potentiel sur l'axe est intéressante pour évaluer les composantes du champ hors de l'axe au moyen de développements limités. Afin de tester ces formules approximatives, nous les avons appliquées à plusieurs systèmes comprenant de multiples électrodes. La comparaison des valeurs approchées aux valeurs rigoureuses montre que l'utilisation de ces formules approchées est tout à fait acceptable pour le calcul des dérivées du potentiel jusqu'à l'ordre 4 y compris. Au-delà de cet ordre, les erreurs par addition deviennent vite trop importantes pour envisager l'emploi de l'approximation.

3. Conclusion. - Le premier type de formules permet le calcul exact des dérivées du potentiel sur l'axe. Après mise en fichier des fonctions de Green, on accède très rapidement à la connaissance du champ en tout point hors de l'axe. Ces formules mettent en évidence l'influence des paramètres géométriques.

Les expressions approchées ne faisant intervenir que des fonctions hyperboliques sont utilisables pour le calcul du potentiel sur l'axe et de ses quatre premières dérivées. Le potentiel et le champ sont donc facilement calculables de cette manière en des points pas trop éloignés de l'axe où les deux premiers termes des développements limités suffisent pour décrire les composantes du champ.

\section{Bibliographie}

[1] Berger, C., Babout, M., C.R. Hebd. Séan. Acad. Sci. B 275 (1972) 333.

[2] Berger, C., Bernard, R., J. Microsc. 20 (1974) 115-126.

[3] Durand, E., Electrostatique, tome II et III (Editions Masson et $\mathrm{Cie)} 1966$

[4] El-Kareh, A. B., El-Kareh, J. C. J., Electron Beams, Lenses and Optics 1 (Academic Press, New York) 1970.

[5] Grivet, P., Electron Optics (Pergamon, Londres) 1965.

[6] Verster, J. L., Philips Res. Rep. 18 (1963) 465.

[7] Natali, S., Di Chio, D., Kuyatt, C. E., J. Res. Nat. Bur. Stand. 76A (1972) 27-35.

[8] Natali, S., Di Chio, D., Kuyatt, C. E., Rev. Sci. Instrum. 43 1 (1972) 80-83.

[9] Natali, S., Di Chio, D., Kuyatt, C. E., Rev. Sci. Instrum. 43 1 (1972) 84-87.
[10] Read, F. H., Adams, A., Soto-Montiel, J. R., J. Phys. E 4 (1971) 625-632.

[11] Adams, A., Read, F. H., J. Phys. E 5 (1972) 150-155.

[12] Adams, A., Read, F. H., J. Phys. E 5 (1972) 156-160.

[13] Read, F. H. et HaRTing, E., Electrostatic Lenses (Elsevier, Amsterdam) 1976, 45-63.

[14] Gallai, S., Szilágyi, M., Period. Polytech. Electr. Eng. 21-3 (1977) 243-247.

[15] Bonjour, P., Revue Phys. Appl. 14 (1979) 533-540.

[16] Bonjour, P., Revue Phys. Appl. 14 (1979) 715-728.

[17] Bertram, S., J. Appl. Phys. 13 (1942) 496-502.

[18] Wanberg, B., Sköllermo, A., J. Electron Spectrosc. Relat. Phenom. 10 (1977) 45. 